\title{
Human Cytomegalovirus and Human Herpesvirus-6 and Wilms Tumor: Is There a Link?
}

\author{
Maryam Kazemi Aghdam ${ }^{1,2}$, Seyed Alireza Nadji ${ }^{3}$, Maliheh Khoddami ${ }^{1,2,{ }^{*}}$ and Mohamad Mahdi \\ Tehrani $^{4}$ \\ ${ }^{1}$ Pediatric Pathology Research Center, Research Institute for Children's Heath, Shahid Beheshti University of Medical Sciences, Tehran, Iran \\ ${ }^{2}$ Department of Pathology, School of Medicine, Shahid Beheshti University of Medical Sciences, Tehran, Iran \\ ${ }^{3}$ Virology Research Center (VRC), National Research Institute of Tuberculosis and Lung Diseases (NRITLD), Shahid Beheshti University of Medical Sciences, Tehran, Iran \\ ${ }^{4}$ Department of Pathology, Hormozgan University of Medical Sciences, Bandarabas, Iran \\ "Corresponding author: Pediatric Pathology Research Center, Research Institute for Children's Heath, Shahid Beheshti University of Medical Sciences, Tehran, Iran;Department \\ of Pathology, School of Medicine, Shahid Beheshti University of Medical Sciences, Tehran, Iran. Email: malihehkhoddami@yahoo.com
}

Received 2020 April 19; Revised 2020 July 18; Accepted 2020 August 05.

\begin{abstract}
Background: Identifying etiologic factors contributing to Wilms tumor (WT) is necessary for its prevention and treatment. Oncogenic viruses cause nearly $20 \%$ of all human cancers. Although trials on preventing virus-caused cancers are complex and difficult, but they are not impossible to conduct. Human Cytomegalovirus (HCMV) and human herpes virus-6 (HHV6) can cause different types of cancers.

Objectives: The current study aimed to investigate whether HCMV and HHV6-DNA are present in patients with WT. This is the first study of this kind in Iran.

Methods: This study was performed on patients with kidney disorders who were referring to Mofid Pediatrics Hospital, Tehran (Iran), during 2010-16. In total, 98 kidney samples (49 patients with WT and 49 normal kidneys (autopsy) and kidneys with benign noninfectious lesions) were investigated to identify HCMV and HHV6-DNA. Qualitative Polymerase Chain reaction (PCR) method and nested polymerase chain reaction (nested-PCR) technique were used to identify HCMV and HHV6, respectively.

Results: No significant difference was found between WT patients and controls concerning the HCMV or HHV6.

Conclusions: Based on the findings, it can be concluded that there is no association between these viruses and WT.
\end{abstract}

Keywords: Human Cytomegalovirus, Human Herpes Virus-6, Polymerase Chain Reaction, Wilms Tumor

\section{Background}

Wilms tumor(WT) or nephroblastoma is the most common childhood malignancy, which is an an embryonal tumor originating from renal precursor cells. There appears to be no appreciable sex predilection. WT mostly occurs in infants, and $50 \%$ of cases are diagnosed before the age of three and $90 \%$ before the age of six years. It rarely occurs as a congenital neoplasm and in adolescents and adults (1).

Identifying etiologic factors contributing to WT is necessary for its prevention and treatment as well as increasing the quality of life of patients. In most cases, no clear cause is known. In addition to genetic factors (1), certain maternal and environmental factors may increase the risk of WT. A study has reported that exposure to pesticides during pregnancy, preterm birth, and high birth weight increases the risk of WT (2). Shrestha et al. (3) also reported a positive association between prenatal exposure to perchloroethylene, formaldehyde, acetaldehyde, and poly- cyclic aromatic hydrocarbons (known carcinogens) during the third trimester and WT.

HHV-6 and HCMV are members of the herpesvirus family that cause life-long persistence infections $(4,5)$. The carcinogenesis of these viruses is also proved by various studies (4, 6-15). As more information becomes available, our knowledge about the role of human herpesviruses in the development of various types of cancer changes.

In addition to some direct oncogenic mechanisms, they can cause the initiation and/or progression of the tumor through influencing the immune system's functions and responses (both immunosuppression and tumorpromoting inflammation) (16).

\section{Objectives}

The current study aimed to investigate the presence of HCMV and HHV6 DNA in patients with WT. Qualitative PCR 
test and nested-PCR technique were used to identify CMV and HHV6, respectively. Detection of viruses in tumors such as WT, could provide useful information for developing treatment protocols, such as target therapy for the virus. According to the best knowledge of the authors, this is the first study of this type in Iran.

\section{Methods}

\subsection{Patients and Controls}

Fifty formalin-fixed, paraffin-embedded (FFPE) tissue samples taken from the kidney of patients with WT were retrieved from the archive of the Department of Pathology of Mofid Pediatrics Hospital, a referral center for pediatrics diseases in Tehran, Iran. Samples were taken from 2010 to 2016. Based on the histological criteria (1), WT was diagnosed by a pediatric pathologist. Fifty age-matched samples were selected as control. These samples included normal kidneys from autopsy specimens and non-tumoral and noninfectious kidney specimens taken from 2010 to 2016.

\subsection{Exclusion Criteria for the Control Group}

Exclusion criteria were as follow: the presence of a tumor, the presence of infectious disease, and history of corticosteroid and immunosuppressive therapy.

University Ethics Committee code: the current study is approved by the Ethics Committee of Shahid Beheshti University of Medical Sciences (code: IR.SBMU.RETECH.REC.1395.95 and IR.SBMU.RETECH.REC.1395.744).

\subsection{Paraffin-Embedded Tissue Section Preparation and DNA Ex- traction}

Archived-tissue sections were deparaffinized and rehydrated with xylene and ethanol solutions. The genome was extracted from the tissue sections according to the RTP $^{\circledR}$ DNA/RNA Virus Mini Kit procedure (Stratec molecular, Berlin, Germany). The extracted nucleic acids were kept in the freezer until upstream processes.

\subsection{Polymerase Chain Reaction (PCR)}

Before CMV and HHV-6 screening, the b-globin gene amplification was performed using the PC03/PC04 and $\mathrm{GH} 20 / \mathrm{PCO} 4$ primer sets to assess the quality of DNA extraction (17). The process was conducted following the Sybr Green Real-time PCR-Melting curve format.

\subsubsection{Screening}

A 116 bp gene region of the envelope glycoprotein $B$ (gpUL55) was used for the identification of CMV genome by the TaqMan real-time PCR method. The primer and probe sequences were: CMV-r ; 5'-AAGTACCCCTATCGCGTGTG-3', CMV-f; 5'-ATGATGCCCTCRTCCARGTC -3', CMV-P ; 5'-FAMTGGCCCAGGGTACGGATCTTATTCG-BHQ1-3' (18). The realtime PCR amplification program was performed at $94^{\circ} \mathrm{C}$ for 10 minutes, followed by denaturation at $94^{\circ} \mathrm{C}$ for $10 \mathrm{sec}$ onds, and annealing and extension at $60^{\circ} \mathrm{C}$ for one minute (in 50 cycles). RT-PCR System CFX-96(BIO-Rad, USA) with HS Prime Taq Premix TaqMan reagent (GENETBIO, Korea) was used for assessing the RT- PCR.

\subsubsection{HHV6 Screening}

HHV-6 genome was identified by a Nested-PCR assay that is described elsewhere (19). The nested PCR was carried out using the main viral capsid protein primer sets amplifying a 214 bp gene region of the virus genome; (HHV1 (outer): 5'-CAATGCTTTTCTAGCCGCCTCTTC-3' and HHV2 (outer): 5'- ACATCTATAATTTTAGACGATCCC-3' and HHV3 (inner): 5'- TTGTGCGGGTCCGTTCCCATCATA-3 and HHV-4 (inner): 5'- TCGGGATAGAAAAACCTAATCCCT).

\subsection{Statistical and Ethical Considerations}

The sample size was calculated using the following formula:

Sample Size $=\frac{Z_{1-\propto / 2}{ }^{2} P(1-P)}{d^{2}}$

$Z_{1-\propto / 2}=1.96 ; d=0.05 ; P=0.075$

Data were analyzed using SPSS version 13.0 software [Statistical Procedures for Social Sciences; Chicago, Illinois, USA]. The Fisher test was used for analyzing the data. A P value $<0.05$ was considered as statistically significant.

To observe confidentially of information, all data were collected unanimously, and reports were only examined by the pathologists. The materials taken from the references are not changed.

\section{Results}

Two samples didn't meet inclusion criteria (one from each group) for PCR; therefore, 49 samples in each group were analyzed. Twenty-seven samples were taken from males and 22 from females. The youngest and oldest patients were 2 months and 10 years, respectively. For the WT patients, HCMV-DNA was detected in three (6.1\%) samples (positive in $6.1 \%$ and negative in 93.9\%). For controls, $H C M V$-DNA was found in five (10.2\%) samples (negative in 
89.8\% and positive in $10.2 \%)$ with a P-value of 0.7 (0.7 > 0.05). The difference between the two groups concerning the HCMV results was not significant. HHV6-DNA was detected in three patients with WT and three samples of the control group ( $6 \%$ positivity, P value $>0.05$ ).

\section{Discussion}

To develop new therapeutic options and/or preventive approaches to cancers, information on etiologic factors are required. Lately, some studies investigated prevention from virus-linked cancers, which would be complex and difficult, but not impossible $(20,21)$. A combination of vaccines and medications that inhibit neoplastic cells from concealing from the immune response is proposed to treat virus-caused cancers (21).

In the current study, as the first of this type in Iran, the PCR technique was used to detect HCMV and HHV6- DNA, and no statistically significant difference was found between the two groups. Therefore, there was no association between WT and these viruses in patients investigated in the current study.

WT is the most common childhood renal tumor (it accounts for nearly $85 \%$ of the kidney tumors), which mostly occurs before the age of six (1). Although the average treatment rate and five-year survival have improved dramatically during recent years, special attention should be paid to the immediate and long-term side effects of WT treatment. Identifying new methods of WT prevention not only is useful to decrease the burden of the disease but also would be a significant progress in developing therapeutic options.

HCMV can contaminate most human tissues and organs, including renal epithelial cells. The model of oncomodulation (progression and spread of the tumorinduced by viral regulatory proteins and non-coding RNA) (4) can be useful to explain the tumorigenicity of HCMV in some, but not all, of the HCMV infected tumors. HCMV is one of the human oncoviruses because of the ability of its gene products to affect many cell functions, such as dysregulation of the cell cycle, immortalization, mutation, and viral genome instability, improved survival, and immune system escape with tumor progression and spread (12). In addition to seroepidemiological facts, HCMV-DNA, messenger RNA(mRNA), and/or antigens are reported in tumor tissues $(4,6)$. A study has reported that $44 \%$ of children with WT and $40 \%$ of patients with neuroblastoma had HCMV antibodies in their sera (7). Wolmer-Solberg and colleagues (11) reported that six neuroblastoma cell lines and all 36 primary neuroblastomas were contaminated with HCMV (HCMV immediate-early protein in $100 \%$, and late protein in 92\%); however, the authors noted that no infectious virus was separated. Considerable reduction in cancer growth and viral protein was observed both in vivo and in vitro after administration of $H C M V$-specific antiviral drugs, suggesting a significant contribution of HCMV in the development of neuroblastoma, as well as the high potential of administering antiviral medication as a therapeutic option in future therapies.

Scheurer et al. (8) investigated 21 glioblastomas (GBM) using the sensitive immunohistochemically (IHC) and in situ hybridization (ISH) techniques and identified HCMVDNA and antigen in 21 cases. They also found the HCMVDNA and antigen in 9 (out of 12) anaplastic gliomas and in 14 (out of 17) low-grade gliomas. IHC showed that $79 \%$ of GBM cells were HCMV-positive and $48 \%$ of cells were positive in lower-grade gliomas, suggesting that HCMV infections may cause and/or facilitate the progression of malignant glial tumors. Also, the nucleic acids of HCMV and/or proteins were identified in all 22 prostatic preneoplastic conditions and neoplasms by IHC, ISH, PCR, and DNA sequencing. Viral proteins were stronger and more frequent in the PIN and basal cell hyperplasia, and had a smaller amount in carcinoma cells (9). Melnick et al. (10), using the IHC, suggested a contributory association between HCMV and mucoepidermoid carcinoma of the salivary gland. In addition, HCMV proteins (IE1-72 and pp65) were identified in $82 \%$ and $78 \%$ colorectal polyps, respectively, and $80 \%$ and $92 \%$ of adenocarcinomas, respectively. The adjacent nontumoral biopsies from the same patients were negative (13).

According to the literature, $H H V-6$ is related to several types of cancers. Gliomas of 88 untreated children were investigated for detecting HHV-6 using the IHC and ISH as well as the nested PCR method and were compared to nonglial neoplasms and normal brain. The nested PCR detected HHV-6U57 in 57\% of tumors (P-value: 0.001), and 61\% of tumors had HHV-6U31 ( $\mathrm{P}=0.019)$. HHV-6U57 was proved in $54 \%$ of tumors using the ISH (P value: 0.021 ), indicating a non-lymphocytic source of $H H V-6$. IHC detected $H H V$ 6A/B gp116/64/54 late antigen in 40\% of tumors (P: 0.013). The IHC positivey was seen in $58 \%$ of low-grade gliomas compared to $19 \%$ positivity in gliomas of higher grade $(\mathrm{P}$ : 0.002 ) and $25 \%$ of nonglial tumors (P-value: 0.001). Colocalization of $H H V-6 \mathrm{~A} / \mathrm{B}$ gp116/64/54 antigen with glial fibrillary acidic protein confirmed the astrocytic derivation (14). Crawford et al. (15) showed a 47\% positivity for HHV-6 U57 Major Capsid Protein (MCP) gene in adult primary and recurrent CNS neoplasms by ISH $(\mathrm{P}=0.001)$ and nested PCR $(\mathrm{P}=0.001)$. Active infection was suggested because $H H V$ $6 \mathrm{~A} / \mathrm{B}$ early ( $\mathrm{p} 41)$ antigen was detected in $24 \%(\mathrm{P}=0.003)$ and late antigen (gp116/64/54) was discovered in 35\% of tumors (P value $=0.002)$ by IHC. The IHC showed that glial tumors are three times more positive for both HHV-6 early and late 
antigens compared to nonglial tumors (15).

$H H V-6$ positivity is also reported in oral squamous cell carcinoma (22) and in skin cancer with a three-time higher risk in basal cell carcinoma (23). By the time writing the present study, there was no study in English Medical literature concerning the association between WT and HHV6.

The current study had limitations, including a small sample size $(n=49)$. In addition, only the presence of the viruses was evaluated, and due to the technological limitations, the etiologic role was not investigated. Studies with a larger sample size with more advanced techniques are recommended to investigate any etiologic role.

\subsection{Conclusions}

HCMV-DNA was identified in only three (6.1\%) WT samples. Five cases in the control group had a CMV virus (10.2\%), which might be a coincidence. However, the difference between WT and control cases concerning the CMV incidence was not statistically significant (P value $0.7>0.05$ ). HHV6DNA was detected in three patients with WT and three patients in the control group (6\% positivity, P value > 0.05). According to the best knowledge of the authors, the current study is the first of this type in Iran, and the findings didn't support the association between HCMV and HHV6 in patients with WT.

\section{Acknowledgments}

The current study that is performed by Dr. Mohamad Mahdi Tehrani and Yasra Mahjoub (under the supervision of Dr. Maliheh Khoddami, Dr. Seyed Alireza Nadji, and Dr. Maryam Kazemi Aghdam) are thesis to achieve the requirements of specialty in Pathology in Pathology at Shahid Beheshti University of Medical Sciences. The authors would like to thank Dr. Saleh Sandoughdaran for the statistical study, Ms. Pooneh Tavakoli and Leila Poos-Ashkan, for doing the PCR, and Ms. Fahimeh Dini for technical assistance.

\section{Footnotes}

Authors' Contribution: Maryam Kazemi Aghdam: Analysis and interpretation of data, revision of the manuscript, and final approval of the version to be published. Seyed Alireza Nadji: Supervising and performing PCR tests, analysis, and interpretation of PCR data. Maliheh Khoddami: Corresponding author, conception and design, supervision, critical revision of the manuscript, and final approval of the version to be published. Mohamad Mahdi Tehrani: Collecting data, drafting of the manuscript.

Conflict of Interests: No conflict of interests to report. Ethical Approval: IR.SBMU.RETECH.REC.1395.95; IR.SBMU.RETECH.REC.1395.744
Funding/Support: The financial support was provided by the Pediatric Pathology Research Center, Research Institute for Children's Health, Shahid Beheshti University of Medical Sciences, Tehran, Iran.

\section{References}

1. McKenney JK. Tumors and tumor like conditions. In: Goldblum JR, Lamps LW, McKenney JK, Myers JL, editors. Rosai and Ackerman's Surgical Pahology. 11th ed. Elsevier Health Sciences; 2018.

2. Chu A, Heck JE, Ribeiro KB, Brennan P, Boffetta P, Buffler P, et al. Wilms' tumour: a systematic review of risk factors and meta-analysis. Paediatr Perinat Epidemiol. 2010;24(5):449-69. doi: 10.1111/j.13653016.2010.01133.x.

3. Shrestha A, Ritz B, Wilhelm M, Qiu J, Cockburn M, Heck JE. Prenatal exposure to air toxics and risk of Wilms' tumor in 0-5 year old children. J Occup Environ Med. 2014;56(6):573-8. doi: 10.1097/JOM.0000000000000167.

4. Michaelis M, Doerr HW, Cinatl Jr J. The story of human cytomegalovirus and cancer: increasing evidence and open questions. Neoplasia. 2009;11(1):1-9. doi: 10.1593/neo.81178.

5. Agut $\mathrm{H}$, Bonnafous P, Gautheret-Dejean A. Laboratory and clinical aspects of human herpesvirus 6 infections. Clin Microbiol Rev. 2015;28(2):313-35. doi:10.1128/CMR.00122-14.

6. Cinatl J, Scholz M, Kotchetkov R, Vogel J, Doerr HW. Molecular mechanisms of the modulatory effects of HCMV infection in tumor cell biology. Trends Molecular Med. 2004;10(1):19-23. doi 10.1016/j.molmed.2003.11.002. [PubMed:14720582].

7. Wertheim P, Voute PA. Neuroblastoma, Wilms' tumor, and cytomegalovirus. J Nat Cancer Institute. 1976;57(3):701-3. doi: 10.1093/jnci/57.3.701. [PubMed: 185403].

8. Scheurer ME, Bondy ML, Aldape KD, Albrecht T, El-Zein R. Detection of human cytomegalovirus in different histological types of gliomas. Acta Neuropathologica. 2008;116(1):79-86. doi: 10.1007/s00401-0080359-1.

9. Samanta M, Harkins L, Klemm K, Britt WJ, Cobbs CS. High prevalence of human cytomegalovirus in prostatic intraepithelial neoplasia and prostatic carcinoma. J Urol. 2003;170(3):998-1002. doi: 10.1097/01.ju.0000080263.46164.97. [PubMed: 12913758].

10. Melnick M, Sedghizadeh PP, Allen CM, Jaskoll T. Human cytomegalovirus and mucoepidermoid carcinoma of salivary glands: cell-specific localization of active viral and oncogenic signaling proteins is confirmatory of a causal relationship. Exp Molecular Pathol. 2012;92(1):118-25. doi: 10.1016/j.yexmp.2011.10.011.

11. Wolmer-Solberg N, Baryawno N, Rahbar A, Fuchs D, Odeberg J, Taher $\mathrm{C}$, et al. Frequent detection of human cytomegalovirus in neuroblas toma: a novel therapeutic target? IntJCancer.2013;133(10):2351-61. doi: 10.1002/ijc.28265.

12. Herbein G. The human cytomegalovirus, from oncomodulation to oncogenesis. Viruses. 2018;10(8):408. doi: 10.3390/v10080408.

13. Harkins L, Volk AL, Samanta M, Mikolaenko I, Britt WJ, Bland KI, et al. Specific localisation of human cytomegalovirus nucleic acids and proteins in human colorectal cancer. Lancet. 2002;360(9345):1557-63. doi:10.1016/S0140-6736(02)11524-8. [PubMed: 12443594]

14. Crawford JR, Santi MR, Thorarinsdottir HK, Cornelison R, Rushing EJ, Zhang $\mathrm{H}$, et al. Detection of human herpesvirus- 6 variants in pediatric brain tumors: association of viral antigen in low grade gliomas.J Clin Virol. 2009;46(1):37-42. doi:10.1007/s11060-009-9908-2.

15. Crawford JR, Santi MR, Cornelison R, Sallinen S, Haapasalo H, MacDonald TJ. Detection of human herpesvirus- 6 in adult central nervous system tumors: predominance of early and late viral antigens in glial tumors. J Neuro-Oncol. 2009;95(1):49-60. doi:10.1016/j.jcv.2009.05.011. [PubMed: 19505845]. [PubMed Central: PMC2749001]. 
16. Alibek K, Baiken Y, Kakpenova A, Mussabekova A, Zhussupbekova $S$, Akan $\mathrm{M}$, et al. Implication of human herpesviruses in oncogenesis through immune evasion and supression. Infect Agents Cancer 2014;9(1):3. doi: 10.1186/1750-9378-9-3.

17. Saiki RK, Bugawan TL, Horn GT, Mullis KB, Erlich HA. Analysis of enzymatically amplified $\beta$-globin and HLA-DQ $\alpha$ DNA with allelespecific oligonucleotide probes. Nature. 1986;324(6093):163-6. doi: 10.1038/324163a0. [PubMed: 3785382].

18. Hernandez AK, Emmons J, Wimmer DB, Payne DA. Cytomegalovirusantigenemia-positive and polymerase-chain-reactionnegative transplant patient. Lab Med. 2008;39(6):341-2. doi: 10.1309/NHKBJ8UFM8HKYJRX.

19. Luiz CR, Machado CM, Canto CL, Christ SC, Pestana JO, Kotton CN, et al. Monitoring for HHV-6 infection after renal transplantation: evaluation of risk factors for sustained viral replication. Transplantation.
2013;95(6):842-6. doi: 10.1097/TP.ob013e3182807ab7.

20. Cohen JI. Epstein-Barr virus vaccines. Clin Transl Immunol. 2015;4(1). e32. doi: $10.1038 /$ cti.2014.27.

21. Smith E. Can we wipe out cancers caused by the Epstein-Barr Virus. Bio Med Central. 2015. Available from: https://blogs.biomedcentral.com/ on-medicine/2015/12/14/can-wipe-cancers-caused-epstein-barrvirus/.

22. Saravani S, Miri-Moghaddam E, Sanadgol N, Kadeh H, Nazeri MR. Human Herpesvirus-6 and Epstein-Barr Virus Infections at Different Histopathological Grades of Oral Squamous Cell Carcinomas. Int JPrevent Med. 2014;5(10):1231-8.

23. Leite JL, Stolf HO, Reis NÁ, Ward LS. Human herpesvirus type 6 and type 1 infection increases susceptibility to nonmelanoma skin tumors. Cancer lett. 2004;224(2):213-9. doi: 10.1016/j.canlet.2004.11.010. [PubMed: 15914272]. 